\title{
Synchronous unilateral parotid gland neoplasms of three different histological types
}

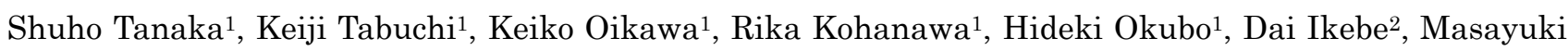
Noguchi $^{2}$, Akira Hara ${ }^{1}$

1. Department of Otolaryngology, Doctoral Program in Functional and Regulatory Medical Sciences, Graduate School of Comprehensive Human Sciences, University of Tsukuba

2. Department of Molecular Pathology, Doctoral Program in Biomolecular and Integrated Medical Sciences, Graduate School of Comprehensive Human Sciences, University of Tsukuba

Address correspondence to Akira Hara

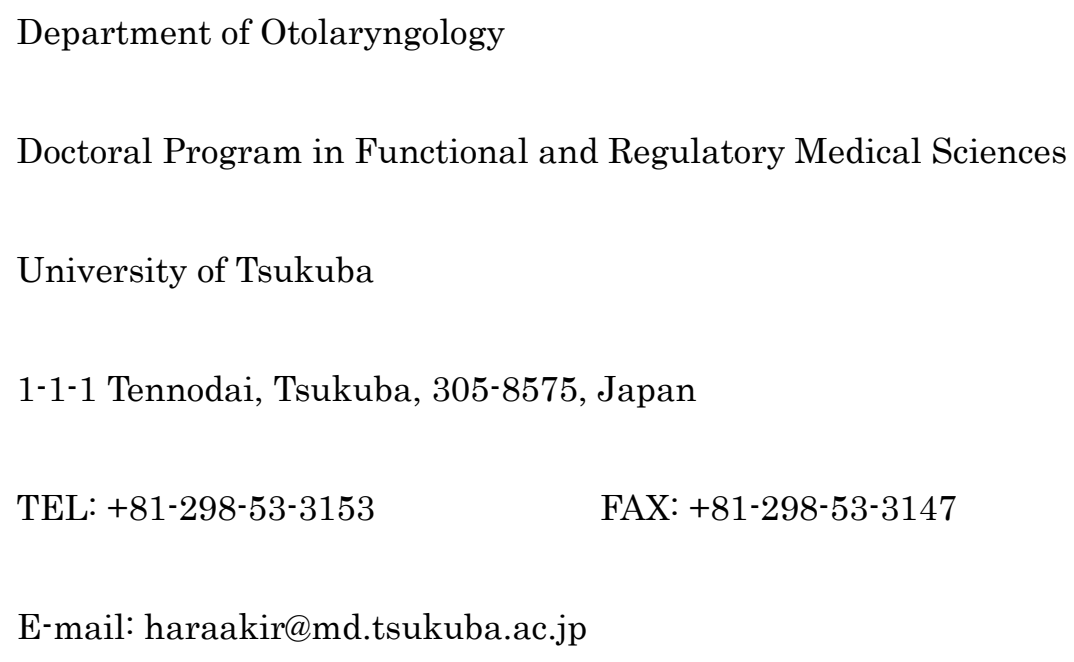

Key words:

synchronous tumors, parotid gland, pleomorphic adenoma, Warthin's tumor, salivary duct carcinoma 


\section{INTRODUCTION}

Salivary gland tumors most frequently arise in a single parotid lesion. Synchronous multifocal parotid neoplasms may occasionally be seen and are usually of "one" histological type, specifically Warthin's tumor. Synchronous parotid tumors of "two" different histological types are rare condition. To our knowledge, there are no previous reports of synchronous unilateral parotid tumors involving "three" or more different histological types. We describe herein a case demonstrating pleomorphic adenoma, Warthin's tumor and salivary duct carcinoma synchronously occurring in the unilateral parotid gland.

\section{CASE REPORT}

In early 1998, a 67-year-old man presented with a right parotid mass. In October 1998, the lesion was operated and diagnosed as a retention cyst at another hospital. He found a mass in the tail of right parotid gland four years after the first operation. A few months later, he noticed another tumor on the anterior edge of the right parotid gland, which gradually increased in size. The patient was first referred to our hospital in December 2003. There was no facial nerve palsy or cervical lymphadenopathy. Based on cytological examination, the lower and anterior tumors were considered pleomorphic adenoma and adenocarcinoma, respectively. Computed tomography (CT) scan and magnetic resonance imaging (MRI) demonstrated that the lower tumor was measured $24 \times 30 \times 35 \mathrm{~mm}$, whereas the anterior tumor measured $18 \times 24 \times 32 \mathrm{~mm}$ and possessed a clear margin(Figure 1. Surgery was performed in February 2003. En bloc removal of the lower tumor and the anterior tumors was achieved with excision of the superficial lobe of the right parotid gland, a part of the masseter muscle and subcutaneous tissues. The buccal branch of the right facial nerve was involved in the anterior tumor and excised along with the tumors. Pathological examination demonstrated that the anterior lesion was salivary duct carcinoma 
(Figure 2A). Salivary duct carcinoma was arranged in nests composed of atypical cells, that formed back to back glands and exhibit cribriform or papillary patterns. The comedo type of necrosis was abundant. Apart from this carcinoma, the inferior lesion consisted of a sharply demarcated pleomorphic adenoma (Figure 2B). This pleomorphic adenoma showed dominant epithelial components intermingled with myxoid, chondroid, and fibrous stroma. In addition, pathological examination disclosed a third distinct tumor near the pleomorphic adenoma. This tiny tumor was composed of a lymphoid matrix containing epithelium-lined cystic space, diagnosed as Warthin's tumor (Figure 2C). These three tumors were each separated from each other. As a result, we found three distinct tumors in the right parotid gland. Postoperative radiotherapy (total 50 Gy) was performed. On subsequent follow-up after one year, there was no sign of recurrence.

\section{DISCUSSION}

Synchronous unilateral or bilateral multifocal tumors of the salivary glands are occasionally seen, accounting for less than $1 \%$ of cases in a series of 877 cases of major salivary gland tumors reported by Foot and Frazell ${ }^{1}$. The majority of these synchronous multiple tumors is composed of one histological type. Warthin's tumor is the most common in multifocal cases, and several cases of multifocal pleomorphic tumor or acinic cell carcinoma have also been reported ${ }^{1}$.

Coexistence of tumors of different histological types is extremely rare in the salivary gland.

Gnepp et al. found such cases comprising less than $0.3 \%$ of all salivary gland neoplasms ${ }^{2}$. The most common combination of synchronous parotid tumors of different histological types is that of Warthin's tumor and pleomorphic adenoma. Alan et al. reviewed that the combination of Warthin's tumor and pleomorphic adenoma was seen in $42 \%$ of those coexisting cases. 
Tanaka and Chen first described a case of synchronous benign and malignant parotid tumors in

$1953^{3}$. Since then only 11 cases of synchronous benign and malignant tumors occurring in the unilateral

parotid gland have been reported. The most common combination of benign and malignant parotid tumors is Warthin's tumor and mucoepidermoid carcinoma ${ }^{4}$. The combination including salivary duct carcinoma is extremely rare, with only one case published previously4.

Only one previous case of three synchronous parotid tumors of different histological types has been reported by Assor ${ }^{5}$. That case demonstrated Warthin's tumor and squamous cell carcinoma in the right parotid gland, and adenocarcinoma in the left parotid gland. Our case had three synchronous but histologically different tumors in the right parotid gland. To our knowledge, this is the first case in the English literature describing synchronous unilateral parotid tumors of three different histological types.

The course and prognosis largely depend on histological types of the neoplasms in cases of synchronous multifocal salivary gland tumors. In our case, one of the components was salivary duct carcinoma. It is a high-grade neoplasm and most commonly seen in the parotid gland. It has been reported that the presence of both local recurrence and distant metastasis to the lung and/or bone is occasionally seen and that patients typically die within 3 years after diagnosis. Postoperative radiotherapy is usually recommended for high-grade malignancies, and was thereby administered to our patient.

\section{CONCLUSION}

We encountered a case of synchronous salivary duct carcinoma, pleomorphic adenoma and Warthin's tumor in the right parotid gland. Simultaneous synchronous occurrence of three different histological parotid tumors is extremely rare, and this is the first case in the English literature. 


\section{REFERENCES}

1. Foote F.W., Frazell E.L. Tumors of the major salivary glands. Cancer. 1953;6: 1065-1133

2. Gnepp D.R., Schroeder W., Heffner D. Synchronous tumors arising in a single major salivary gland. Cancer. 1989;63:1219-1224

3. Tanaka N, Chen W.C. A case of bilateral papillary cystadenoma lymphomatosum (Warthin's tumor) of the parotid complicated with a mucoepidermoid tumor. Jpn J Cancer Res. 1953;144:229-231

4. Curry J.L., Petruzzelli G.J., McClatchey K.D., et al. Synchronous benign and malignant salivary gland tumors in ipsilateral glands: a report of two cases and a review of literature. Head Neck. 2002;3:301-306

5. Assor D. Bilateral carcinoma of the parotid, one cancer arising in a Warthin's tumor. Am $J$ Clin Patho. 1974;61:270-274 
Legends

Figure 1

A: enhanced CT scan of the parotid gland. Mild heterogeneous enhancement was seen in both tumors (arrow heads).

B and C: T1-weighed (B) and T2-weighed (C) MRI showing that both tumors had clear margins (arrow heads).

Figure 2

A: Salivary duct carcinoma (x100) exhibited cribriform or papillary patterns. The comedo type of necrosis is abundant.

B: Pleomorphic adenoma (x100) showed dominant epithelilal components intermingled with myxoid, chondroid, and fibrous stroma.

C: Warthin's tumor (x100) composed of a lymphoid matrix containing epithelium-lined cystic space. 
A

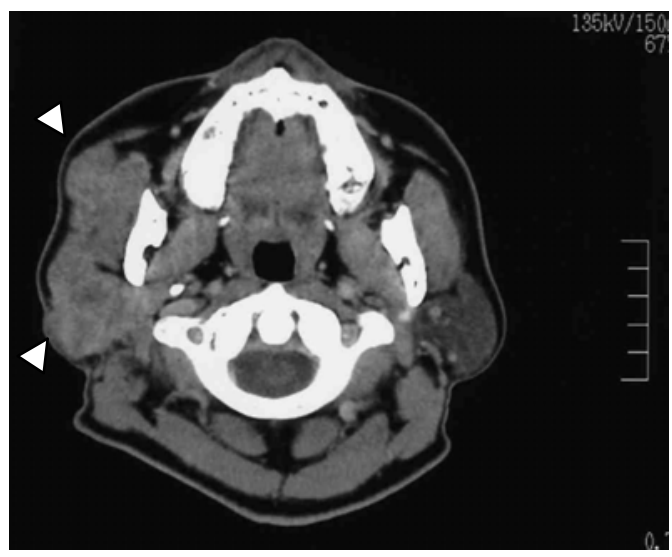

B

C
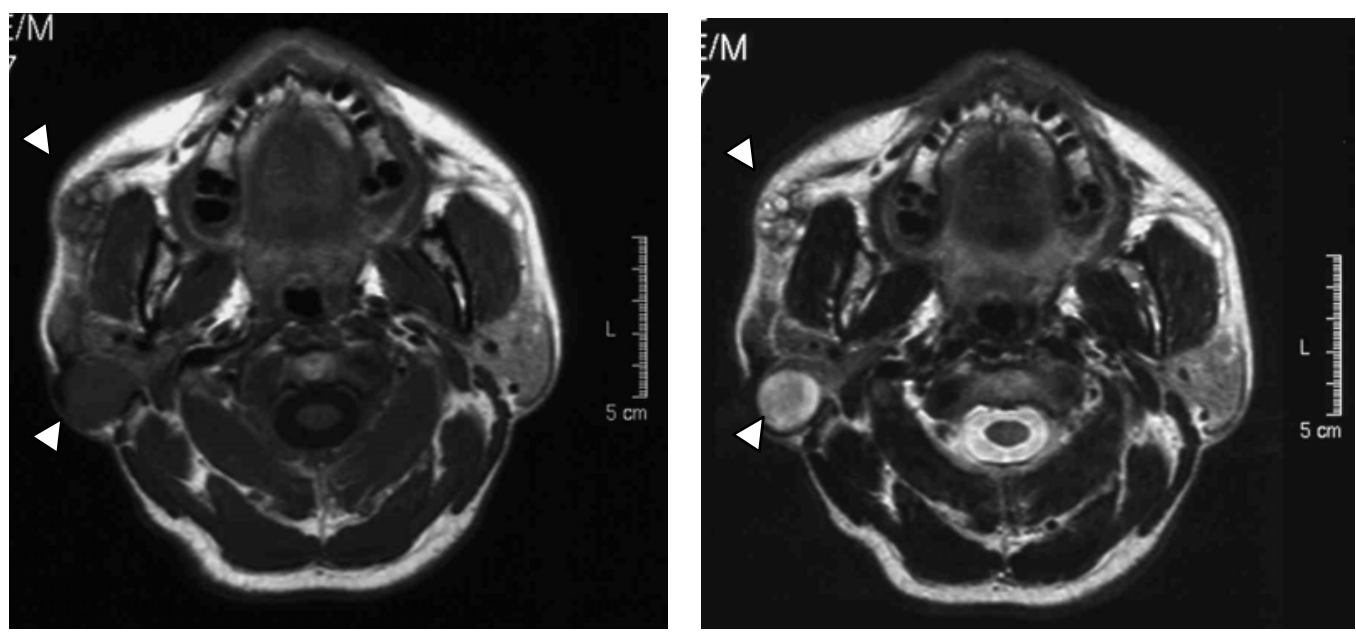

Fig.1 


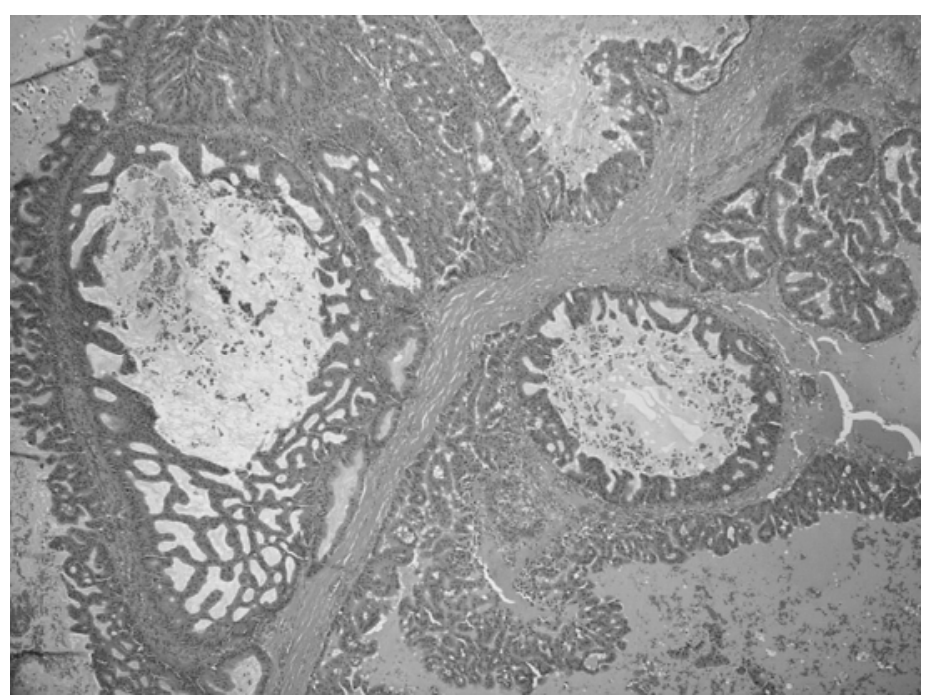

B

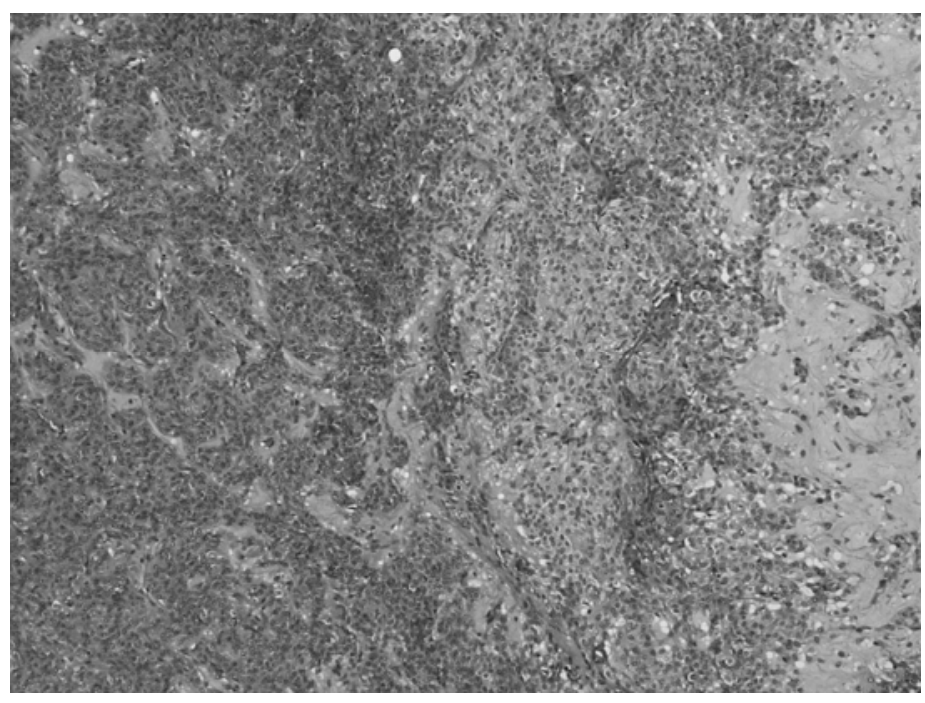

C

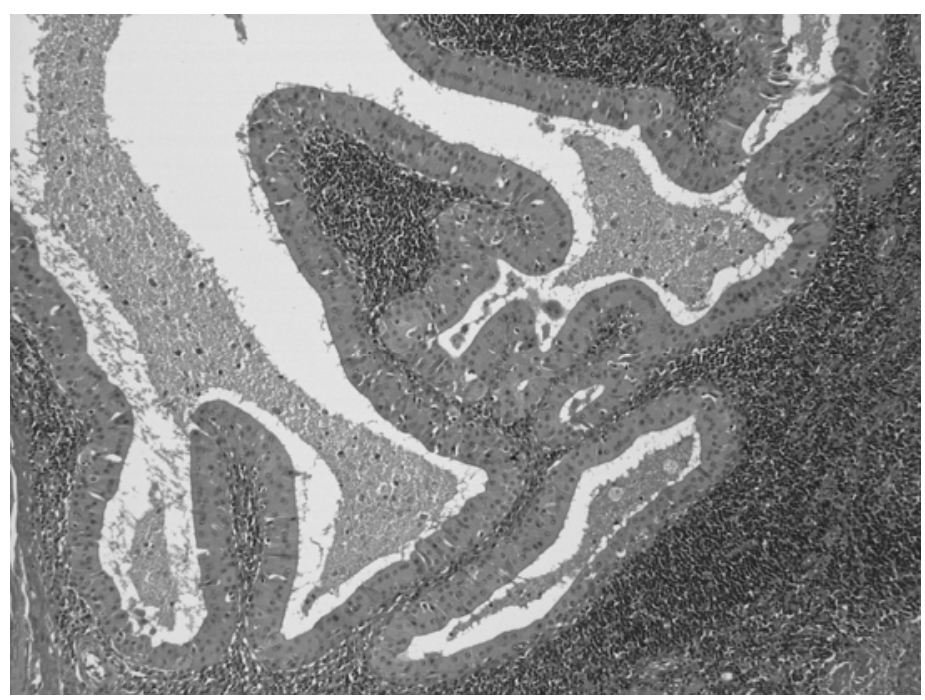

Fig.2 